\title{
Analysis of in vivo turnover of tau in a mouse model of tauopathy
}

\author{
Kaoru Yamada', Tirth K. Patel ${ }^{2}$, Katja Hochgräfe ${ }^{3}$, Thomas E. Mahan², Hong Jiang ${ }^{2}$, Floy R. Stewart ${ }^{2}$, \\ Eva-Maria Mandelkow ${ }^{3,4,5}$ and David M. Holtzman ${ }^{2 *}$
}

\begin{abstract}
Background: Intracellular accumulation of tau as neurofibrillary tangles (NFTs) is the hallmark of Alzheimer's disease (AD) as well as in other tauopathies. Tau is present not only in the cytoplasm but also in the extracellular space such as cerebrospinal fluid (CSF) and brain interstitial fluid (ISF). Although clearance is one critical parameter leading to such intracellular/extracellular tau accumulation, in vivo turnover of tau has not been well characterized. The current study has attempted to precisely determine in vivo turnover rates of tau utilizing tet-off regulatable mice. In particular, we assessed intracellular tau and extracellular tau, soluble tau, insoluble tau and phosphorylated tau at certain sites utilizing a combination of in vivo microdialysis, biochemical analysis and specific ELISAs recognizing each species. To examine the effect of a tauopathy-associated mutation on tau clearance, half-lives of various tau species were compared between the mice with a FTDP-17 mutation that induces $\beta$-sheet formation, $\Delta K 280$ mutation (pro-aggregant mice) and control mice with additional $\beta$-sheet breaking mutations (anti-aggregant mice).

Results: Here we report that tau is metabolized at much slower turnover rates in vivo than in cell culture. We found that insoluble tau in pro-aggregant mice had a significantly slower half-life $\left(\mathrm{t}_{1 / 2}=\sim 34.2\right.$ days) than soluble tau ( $\mathrm{t}_{1 / 2}=\sim 9.7$ days). In contrast, soluble tau phosphorylated in the proline rich region was cleared faster than total soluble tau. When comparing pro-aggregant mice to anti-agregant mice, turnover rates of soluble tau species were not significantly different.

Conclusions: The current study provides a comprehensive description of in vivo turnover of various tau species present in mice that express human tau. The turnover rate of soluble tau was not significantly altered between pro-aggregant mice and anti-aggregant mice. This suggests that altered conformation by $\Delta K 280$ does not have a major impact on clearance pathways for soluble tau. In contrast, different tau species displayed different half-lives. Turnover was significantly delayed for insoluble tau whereas it was accelerated for soluble tau phosphorylated in the proline rich region. These differences in susceptibilities to clearance suggest that aggregation and phosphorylation influences tau clearance which may be important in tau pathogenesis.
\end{abstract}

Keywords: Alzheimer's disease, Clearance, Half-life, Extracellular tau, Tauopathy model

\section{Background}

Abnormal tau aggregates as NFTs are common pathological hallmarks in a set of neurodegenerative diseases called tauopathies including $\mathrm{AD}$, progressive supranuclear palsy, corticobasal degeneration, and certain forms of frontotemporal lobar dementia. Tau is physiologically highly soluble, however under pathological

\footnotetext{
*Correspondence: holtzman@neuro.wustl.edu

${ }^{2}$ Department of Neurology, Hope Center for Neurological Disorders, Knight Alzheimer's Disease Research Center, Washington University School of Medicine, St. Louis, Missouri, 63110, USA

Full list of author information is available at the end of the article
}

conditions, it changes its conformation to one with a high $\beta$-sheet content, undergoes hyperphosphorylation and forms aggregates including filaments and NFTs in the cytoplasm.

NFTs are formed intracellularly, however, extracellular tau also has pathological significance. For example, tau in CSF is elevated in individuals with AD and preclinical $\mathrm{AD}$. In addition, recent studies have demonstrated that tau pathology may be transmitted from neuron to neuron via the extracellular space [1-5].

Growing evidence suggests that tau contributes to neuronal dysfunction. Chronic inhibition of tau expression in 
various mouse models protects them against cognitive impairments [6-8]. This leads to the idea that lowering tau production may be a potential therapeutic strategy. Since clearance pathways mediate tau protein levels, the development of tau-lowering therapy would benefit from a better understanding of in vivo turnover of tau. While a number of studies have focused on how tau is metabolized in cultured cells [9-14], surprisingly little is known about in vivo clearance of tau, especially pathologically relevant tau species that accumulate in the tauopathies such as aggregated forms of tau, extracellular tau, and phosphorylated tau. Therefore, an analysis of the in vivo turnover rate of tau in mice that develop tauopathy will not only deepen our understanding of tau clearance at the systemic level but also assist in interpreting tau changes in the clinical setting.

In order to directly address this issue, we set out to evaluate in vivo turnover rates of tau in animal models that accumulate pathogenic tau species. In this study, we utilized tet-off tau transgenic mice called pro-aggregant mice, which express a regulatable human $2 \mathrm{~N} 4 \mathrm{R}$ tau cDNA with a $\Delta \mathrm{K} 280$ tau mutation. These mice develop agedependent tau aggregation $[6,15,16]$. We examined turnover rates of intracellular soluble tau, insoluble tau, phosphorylated tau and extracellular soluble tau by coupling biochemical analysis and in vivo microdialysis with specific ELISAs recognizing different tau species. Here we report that in vivo turnover rates of tau were significantly longer than in cell culture. Susceptibility to clearance varies among different tau species. Intracellular insoluble tau displayed a significantly longer half-life in pro-aggregant mice and soluble tau phosphorylated in the proline rich region showed faster turnover rates than total soluble tau. When turnover rates of soluble tau were compared between proaggregant mice and aged matched control mice with a $\beta$-sheet breaking mutation in tau called anti-aggregant mice [17], significant differences were not observed.

\section{Results and discussion}

\section{Strategy to investigate turnover of intracellular soluble} tau, insoluble tau, phosphorylated tau and extracellular tau in tet-off regulatable tau transgenic mice

To examine turnover rates of various tau species in vivo, we utilized tet-off tau transgenic mice, called pro-aggregant mice $[6,15,16]$. Pro-aggregant mice express a $2 \mathrm{~N} 4 \mathrm{R}$ isoform of human tau with a $\Delta \mathrm{K} 280$ mutation, which is found in a family with FTDP-17 [18] (Fig. 1a). This mutation modifies MAPT splicing leading to an increase of three repeat isoform, however four repeat isoform with this $\Delta K 280$ mutation is still produced $[18,19]$, which enhances propensity of tau to form $\beta$-sheet structure. In these mice, tau changes its conformation to a form containing high $\beta$-sheet content, forms insoluble aggregates and undergoes hyperphosphorylation, which allows us to assess various pathologically relevant tau species. Although the 16-17 month old mice that we utilized in this study had not yet developed pronounced NFTs at this age, they have detergent insoluble tau [16] and display synapse loss and cognitive decline, which can be reversed when tau expression is suppressed [6].

The expression of human tau in these mice can be specifically switched off by doxycycline. Therefore the rate at which tau steady-state levels decline following doxycycline treatment will reflect its half-life. In addition, the expression of human tau is kept intentionally low to avoid disturbance of endogenous metabolism in these mice.

The outline of our experimental design is illustrated in Fig. 1. Tau is present not only in the intracellular compartment but also in the extracellular space. To distinguish clearance of extracellular tau from intracellular tau, microdialysis was performed in the hippocampus during doxycycline treatment prior to brain collection (Fig. 1b, c). Since the microdialysis technique works on the principle of the diffusion of molecules in solution across a semi-permeable membrane, microdialysis samples contain extracellular soluble tau in ISF which is less than $1,000 \mathrm{kDa}$, the molecular weight cut-off of the probes [20].

To examine intracellular tau, hippocampal homogenates were prepared at the end of doxycycline treatment (Fig. 1b, c). Brain samples include both intracellular and extracellular proteins. However tau is predominantly present intracellularly [21], thus tau in hippocampal homogenates represents mostly intracellular tau (Fig. 1c). The hippocampus was immediately dissected after microdialysis experiments and biochemically processed by serial extraction using reassembly buffer (RAB), RIPA, $70 \%$ formic acid to obtain soluble and insoluble intracellular fractions (Fig. 1b, c). Tau levels were quantified in these fractions (We defined each fraction as follows; ISF = extracellular soluble fraction, hippocampus soluble fraction = intracellular soluble fraction, hippocampus insoluble fraction = intracellular insoluble fraction) by ELISA (Fig. 1c). We utilized a combination of Tau 5 and biotinylated HT7 (HT7B) antibodies to detect human tau and HJ9.2 and biotinylated HJ8.7 (HJ8.7B) antibodies to detect murine tau respectively. We first checked the specificity of these ELISAs using brain homogenates from hTau mice, which express human tau on a murine tau knockout background along with wildtype and tau knockout mice as controls [22]. Tau5/ HT7B ELISA detected tau only in brain homogenates of hTau mice (Fig. 1d). In contrast, the HJ9.2/HJ8.7B ELISA only detected tau in wildtype mice (Fig. 1e). This demonstates that our assays are highly specific to 


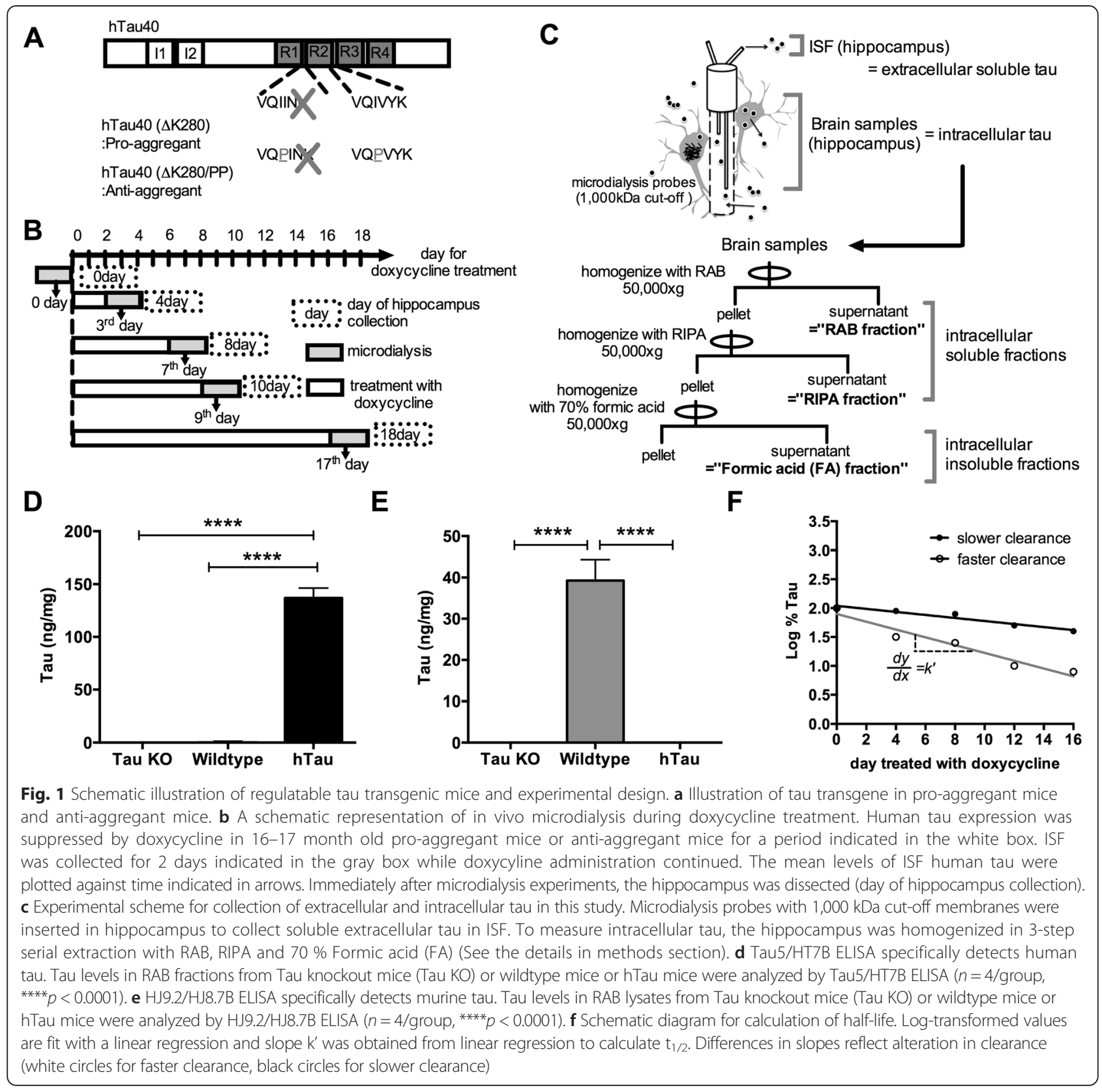

human or murine tau. After tau levels were measured by ELISA, log-transformed tau levels were plotted over days treated with doxycycline along with a linear regression curve and turnover rates were determined by analyzing the slope as previously described [20, 23] (Fig. 1f).

Turnover of soluble tau in pro-aggregant mice and antiaggregant mice

By using hippocampal soluble fractions, we first investigated turnover rates of intracellular soluble tau in 16-17 month old pro-aggregant mice. In these fractions, doxycycline specifically reduced human but not murine tau as expected (Fig. 2a). A linear regression of semi-log plot (Fig. 2c) revealed that the estimated half-life of intracellular soluble tau in pro-aggregant mice was 9.7 days (Table 1 ). The half-life of tau determined in cell culture ranges from $\sim 5$ to $60 \mathrm{~h}[9-12,24]$. The significantly longer half-life of tau indicates that tau metabolism is different between cells and the in vivo environment.

To examine clearance of extracellular tau, in vivo microdialysis was performed in the pro-aggregant mice. Doxycycline treated resulted in a trend of reduction in human tau with no change in ISF lactate (Fig. 2b). The half-life of extracellular tau in pro- 


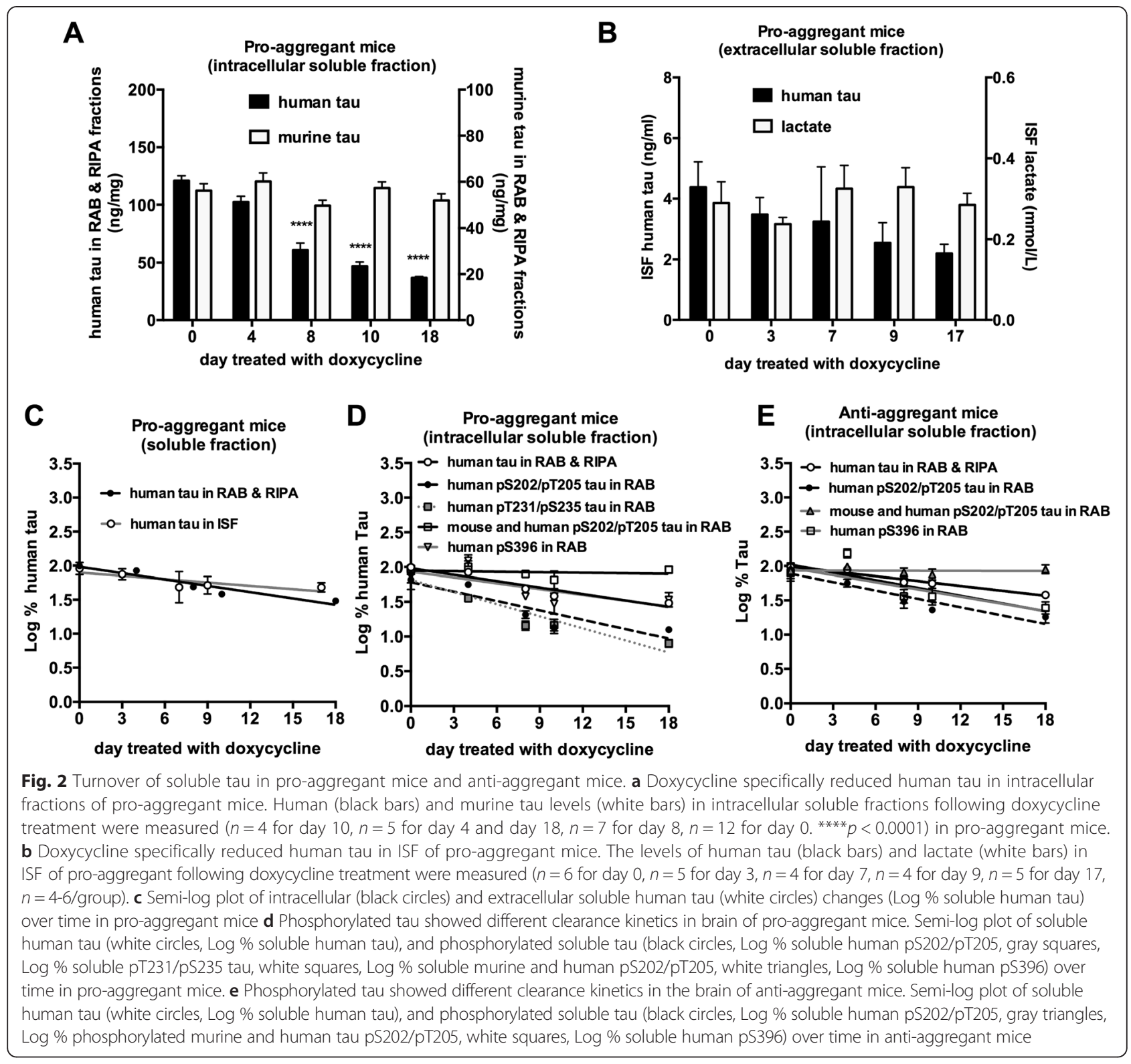

aggregant mice was 17.3 days (Table 1). Although a trend was seen suggesting that extracellular soluble tau has a longer half-life than intracellular soluble tau (Table 1, intracellular soluble vs. extracellular soluble, $p=0.06$ ), turnover rates were not significantly different (Fig. 2c).

Prior studies have linked dysfunction in protein metabolism with disease-associated mutations. For example, TDP-43 mutations linked to amyotrophic lateral sclerosis exhibit a longer half-life [25]. Alpha-synuclein with an A53T mutation associated with familial Parkinson's disease also has increased stability [26]. It is unknown whether tau mutations could also have a similar effect on metabolism. In order to ask whether a longer halflife of tau in pro-aggregant mice is due to the conformational change driven by the $\Delta \mathrm{K} 280$ mutation, turnover of tau in pro-aggregant mice was compared with 16-17 month old anti-aggregant mice where tau cannot form a $\beta$-sheet structure due to additional double proline mutations. There was no significant difference in half-lives of both intracellular soluble tau and extracellular soluble tau between pro-aggregant mice and anti-aggregant mice (Table 2). This suggests that altered conformation by the $\Delta \mathrm{K} 280$ mutation does not have a profound effect on clearance of soluble tau. Importantly, in vivo half-lives of intracellular soluble tau determined for pro-aggregant mice and anti-aggregant mice are comparable to the half-life of endogenous murine tau in brain estimated by stable-isotope labeling [27]. This supports the idea that longer half-life 
Table 1 Half-lives of various tau species in pro-aggregant mice and anti-aggregant mice

\begin{tabular}{|c|c|c|}
\hline \multicolumn{3}{|l|}{ Pro-aggregant mice } \\
\hline & half-life $\left(\mathrm{t}_{1 / 2}\right.$, day) & $R^{2}$ \\
\hline Intracellular soluble & 9.7 & 0.91 \\
\hline Extracellular soluble & 17.3 & 0.7 \\
\hline$P$ & & 0.06 \\
\hline intracellular soluble & 9.7 & 0.91 \\
\hline intracellular soluble pS202/pT205 & 5.7 & 0.8 \\
\hline$P$ & & 0.12 \\
\hline intracellular soluble & 9.7 & 0.91 \\
\hline intracellular soluble pT231/pS235 & 5.2 & 0.88 \\
\hline$P$ & & $<0.0001$ \\
\hline intracellular soluble & 9.7 & 0.91 \\
\hline intracellular insoluble & 34.2 & 0.43 \\
\hline$P$ & & $<0.0001$ \\
\hline intracellular soluble & 9.7 & 0.91 \\
\hline intracellular soluble pS396 & 9.8 & 0.51 \\
\hline$P$ & & 0.52 \\
\hline \multicolumn{3}{|l|}{ Anti-aggregant mice } \\
\hline & half-life $\left(\mathrm{t}_{1 / 2}\right.$, day) & $R^{2}$ \\
\hline Intracellular soluble & 11.1 & 0.96 \\
\hline Extracellular soluble & 10.9 & 0.91 \\
\hline \multirow[t]{2}{*}{$P$} & & 0.44 \\
\hline & half-life $\left(\mathrm{t}_{1 / 2}\right.$, day) & $R^{2}$ \\
\hline intracellular soluble & 11.1 & 0.96 \\
\hline intracellular soluble pS202/pT205 & 7.5 & 0.89 \\
\hline \multirow[t]{2}{*}{$P$} & & 0.022 \\
\hline & half-life $\left(\mathrm{t}_{1 / 2}\right.$, day $)$ & $R^{2}$ \\
\hline intracellular soluble & 11.1 & 0.96 \\
\hline intracellular soluble pS396 & 7.6 & 0.64 \\
\hline$P$ & & 0.24 \\
\hline
\end{tabular}

of tau in tet-off tau transgenic mice is unlikely to derive from technical differences or over-expression.

Aberrant phosphorylation of tau is one of the key features of AD. To see whether phosphorylation alters turnover of soluble tau, we investigated the half-life of human phosphorylated tau at S202/T205 and T231/ S235 by using human tau specific HJ8.5 antibody [28] along with phospho-specific antibodies AT8 and AT180 antibodies in ELISAs. We found that intracellular soluble tau phosphorylated at S202/T205 and T231/S235 displayed faster turnover rates than total soluble tau in pro-aggregant mice (Fig. 2d). The half-life of tau phosphorylated at S202/T205 and T231/S235 was 5.7 days and 5.2 days respectively (Table 1 ). The turnover rate of phosphorylated human tau at S202/T205 was also
Table 2 The comparison of half-life of tau between proaggregant mice and anti-aggregant mice

\begin{tabular}{|c|c|c|}
\hline \multicolumn{3}{|c|}{ Intracellular soluble tau } \\
\hline & half-life $\left(\mathrm{t}_{1 / 2}\right.$, day) & $R^{2}$ \\
\hline Pro-aggregant & 9.7 & 0.91 \\
\hline Anti-aggregant & 11.1 & 0.96 \\
\hline$P$ & & 0.06 \\
\hline \multicolumn{3}{|c|}{ Extracellular soluble tau } \\
\hline & half-life $\left(\mathrm{t}_{1 / 2}\right.$, day) & $\mathrm{R}^{2}$ \\
\hline Pro-aggregant & 17.3 & 0.7 \\
\hline Anti-aggregant & 10.9 & 0.91 \\
\hline$P$ & & 0.25 \\
\hline \multicolumn{3}{|c|}{ Intracellular soluble pS202/pT205 tau } \\
\hline & half-life $\left(\mathrm{t}_{1 / 2}\right.$, day) & $R^{2}$ \\
\hline Pro-aggregant & 5.7 & 0.8 \\
\hline Anti-aggregant & 7.5 & 0.89 \\
\hline P & & 0.64 \\
\hline \multicolumn{3}{|c|}{ Intracellular soluble pS396 tau } \\
\hline & half-life $\left(\mathrm{t}_{1 / 2}\right.$, day) & $R^{2}$ \\
\hline Pro-aggregant & 9.8 & 0.51 \\
\hline Anti-aggregant & 7.6 & 0.64 \\
\hline$P$ & & 0.45 \\
\hline
\end{tabular}

faster in anti-aggregant mice and it was similar to that seen in pro-aggregant mice (Fig. 2e, Tables 1 and 2). In order to examine whether this faster turnover is indeed due to faster clearance, we utilized an ELISA that equally detects both murine and human phosphorylated tau using the HJ8.7 antibody (which recognizes both murine and human tau) instead of the human tau specific HJ8.5 antibody. We did not observe faster decline of phosphorylated tau in both pro-aggregant mice and anti-aggregant mice using this assay, suggesting that faster turnover of phosphorylated human tau is likely due to faster clearance not due to faster turnover of the phosphate group itself (Fig. 2e). Both S202/T205 and T231/S235 are located in the proline rich region of tau. In order to examine the effect of phosphorylation in the carboxyl terminal region of tau, we also investigated half-life of human tau phosphorylated at S396. The half-life of tau phosphorylated at S396 in pro-aggregant mice and anti-aggregant mice was 9.8 days and 7.6 days respectively (Fig. 2d, e) which is not as short as the half-life of human tau phosphorylated at the S202/ T205 and T231/S235 sites. Taken together, this data suggests that phosphorylation of tau at some sites differentially alters susceptibility of soluble tau to clearance. In 
fact, it is consistent with the studies showing that some species of phosphorylated tau is preferentially degraded in certain contexts $[11,13]$.

\section{Turnover of intracellular insoluble tau in pro-aggregant mice}

The comparison between pro-aggregant mice and antiaggregant mice revealed that the turnover rate of soluble tau was not different between these mice. Thus we asked whether turnover of tau is altered when it forms insoluble aggregates. Doxycycline resulted in a decrease in human tau in the detergent insoluble fractions without changing murine endogenous tau in pro-aggregant mice although the decrease was not quite statistically significant (Fig. 3a). The estimated turnover rate of insoluble tau was 34.2 days, suggesting that insoluble tau is cleared significantly more slowly than soluble tau (Fig. $3 \mathrm{~b}$ and Table 1). Tau in insoluble fractions likely represents tau oligomers, fibrils, and other $\beta$-sheet enriched assemblies not present in anti-aggregant mice. Thus the data indicates although soluble tau is normally metabolized in pro-aggregant mice, once it forms insoluble structures, it acquires relative resistance to degradation and clearance. Interestingly though, even some clearance of insoluble material occurs suggesting that endogenous mechanisms for aggregate clearance are present.

\section{Conclusions}

In this study, we examined turnover rates of various tau species. We found that in vivo half-life of tau is significantly longer than tau half-life previously reported in vitro studies. Therefore, reducing tau production therapeutically will take an appreciable period of time to reduce tau protein levels, especially pre-existing tau aggregates than previously predicted from cell culture studies.

We also asked whether the $\Delta \mathrm{K} 280$ mutation influences turnover of tau. The half-life of soluble tau was not significantly altered when comparing pro-aggregant to anti-aggregant mice. The data suggests that despite the propensity to altered conformation, the $\Delta \mathrm{K} 280 \mathrm{mu}$ tation does not lead to a profound effect on clearance of soluble tau. Further studies will be needed to examine whether other tau mutations have an effect on soluble tau clearance.

The mechanistic reason to explain differences in the half-life of various tau species is not known. Furthers studies to understand the mechanisms causing differences in in vivo clearance of tau will be helpful. In addition, the systems that clear extracellular tau in the ISF or CSF will be particularly important to explore since they differ completely from the intracellular degradation pathways important for intracellular tau degradation such as the proteasomal or autophagy systems. Although it was not statistically significant with the number of mice we had, there was a trend that extracellular soluble tau has a longer half-life than intracellular soluble tau in pro-aggregant mice $\left(\mathrm{t}_{1 / 2}=9.7\right.$ day for intracellular soluble tau, $\mathrm{t}_{1 / 2}=17.3$ day for extracellular soluble tau, $p=0.06$, Table 1 ). This trend was not observed in anti-aggregant mice $\left(\mathrm{t}_{1 / 2}=\right.$ 11.1 day for intracellular soluble tau, $\mathrm{t}_{1 / 2}=10.9$ day for extracellular soluble tau, $p=0.44$, Table 1) [20]. Although the increasing trend in half-life of extracellular tau in pro-aggregant mice should be further examined
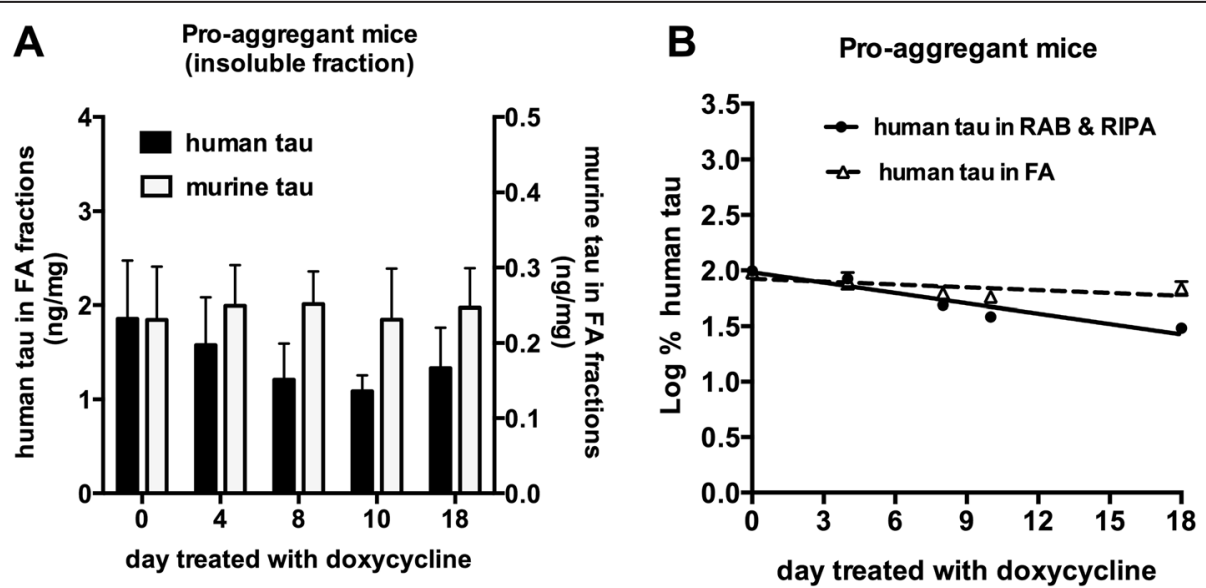

Fig. 3 Turnover of intracellular insoluble tau in pro-aggregant mice. a Doxycycline specifically reduced human tau in FA fractions of pro-aggregant mice. Human (black bars) and murine tau levels (white bars) in detergent insoluble fractions of pro-aggregant mice following doxycycline treatment were measured. ( $n=4$ for day 10, $n=5$ forday 4 and day $18, n=7$ for day $8, n=12$ for day 0 .). b Insoluble tau showed slower turnover than soluble tau in brain. Semi-log plot of soluble (black circles) and detergent insoluble human (white triangles) tau (Log \% human tau) over time in pro-aggregant 
with a larger number of mice, one possibility for this trend might be that turnover of extracellular tau is differentially influenced by the presence of tau aggregates, due to an equilibrium between ISF tau and tau aggregates. Such an equilibrium was suggested by our group previously using P301S human tau transgenic mice [21].

Although we are not currently able to detect soluble tau oligomers or aggregates in the ISF by microdialysis, it will be important to understand clearance mechanisms of such extracellular tau species that facilitate the spreading of tau pathology from cell to cell in the brain if present. Besides its role as a diagnostic biomarker in $\mathrm{AD}$, soluble tau in CSF is being evaluated as an endpoint to validate the disease-modifying effects in various clinical trials for AD. In addition to tau released from dying or degenerating neurons, the long half-life of extracellular tau actively released from neurons may provide some important new insights to consider as to what might be leading to elevated extracellular tau as well as tau species that may mediate intercellular tau spreading.

\section{Methods}

\section{Animals}

All animal experiments were performed and approved in accordance with guidelines established by the Animal Studies Committee at Washington University. Regulatable transgenic mice expressing human full-length tau cDNA (Tau 2N4R) with mutations $\Delta$ K280/PP (I277P, I308P) (anti-aggregant mice) and mice expressing human full-length tau cDNA (Tau 2N4R) with a $\Delta K 280$ mutation (pro-aggregant mice) on $\mathrm{C} 57 \mathrm{BL} / 6 \mathrm{~J}$ background of both sexes were utilized. The mice were screened ahead of experiments by quantifying transgene expression via the co-expressed luciferase detected by bioluminescence imaging $[6,15]$. P301S transgenic mice (line PS19) which overexpress P301S human T34 isoform tau (1N4R) on a B6C3 background were used to make brain homogenates as a standard for phosphorylated tau measurements [29]. Three month old tau knockout mice were obtained from the Jackson laboratory [30]. Five to nine month old wildtype littermates of P301S transgenic mice were used as wildtype controls. 11.5-12.5 month old hTau mice which express wildtype human tau on a tau knockout background were also used [22].

\section{in vivo microdialysis}

in vivo microdialysis with a $1,000 \mathrm{kDa}$ molecular weight cut-off probe to assess ISF tau levels from awake and freely moving mice was performed as previously described [20].
Briefly, a guide cannula (Eicom microdialysis) was stereotactically implanted in the left hippocampus under isoflurane anesthesia, and cemented. After implantation of the cannula and dummy probes (Eicom microdialysis), mice were habituated to microdialysis cages for one more day. After this recovery period, a 2-mm 1,000-kD cut-off Atmos ${ }^{\mathrm{LM}}$ microdialysis probe (Eicom) was inserted through the guide cannula. As a perfusion buffer, $25 \%$ human albumin solution (Gemini Bio Inc.) was diluted to $4 \%$ with artificial CSF (aCSF; $1.3 \mathrm{mM} \mathrm{CaCl}_{2}$, $1.2 \mathrm{mM} \mathrm{MgSO}, 3 \mathrm{mM} \mathrm{KCl}, 0.4 \mathrm{mM} \mathrm{KH_{2 }} \mathrm{PO}_{4}, 25 \mathrm{mM}$ $\mathrm{NaHCO}_{3}$, and $122 \mathrm{mM} \mathrm{NaCl}, \mathrm{pH}$ 7.35) on the day of use and filtered through a $0.1 \mu \mathrm{m}$ membrane. A probe was connected to a microdialysis peristaltic pump with two channels (MAB20; SciPro), which was operated in a push-pull mode. Before microdialysis sample collection, a pump was run at the maximum flow rate for at least $1 \mathrm{~h}$ and then the flow rate was switched to $0.5 \mu \mathrm{l} / \mathrm{min}$. To avoid tissue damage, the experimental window was set from 6 to $48 \mathrm{~h}$ after probe implantation. ISF samples were collected in a refrigerated fraction collector (SciPro) and analyzed by ELISA.

\section{Switch off experiments}

Sixteen to seventeen month old pro-aggregant or antiaggregant mice were randomly divided into 5 groups. One cohort of mice was not exposed to doxycyclinecontaining food pellets $(200 \mathrm{mg} / \mathrm{kg}$, Bio-serv $)$ and ISF was collected for 2 days ( 0 day). Other cohorts of mice received doxycycline-containing food pellets for 4,8 , 10 , or 18 days respectively prior to microdialysis. On the 2nd, 6th, 8th, or 16th day, in vivo microdialysis was performed and ISF was collected for additional 2 days. During microdialysis, mice were given doxycyclinecontaining food pellets. Mean levels of ISF human tau, lactate were plotted from the 3rd, 7th, 9th, or 17th day. On the 4th, 8th, 10th, or 18th day, immediately after the end of microdialysis experiments, brains were collected. Values of \% human tau in brain or ISF and \% lactate in ISF were normalized by the mean concentration present in the day 0 groups.

\section{Brain extraction}

Mice were transcardially perfused with heparin-PBS and brains dissected and kept at $-80{ }^{\circ} \mathrm{C}$ until analyzed. Hippocampus was weighed and homogenized in welldefined 3-step serial extraction protocol described previously [16, 21, 28, 29]. Hippocampus was first homogenized with RAB buffer containing $(100 \mathrm{mM}$ MES, $1 \mathrm{mM}$ EDTA, $0.5 \mathrm{mM} \mathrm{MgSO}_{4}, 750 \mathrm{mM} \mathrm{NaCl}$, $20 \mathrm{mM} \mathrm{NaF}, 1 \mathrm{mM} \mathrm{Na} \mathrm{VO}_{4}$, supplemented by protease inhibitors (Complete, Roche) and phosphatase inhibitors (PhosSTOP, Roche). After centrifugation at 
$50,000 \times \mathrm{g}$ for $20 \mathrm{~min}$, the $\mathrm{RAB}$ insoluble pellet was solubilized with RIPA buffer $(150 \mathrm{mM} \mathrm{NaCl}, 50 \mathrm{mM}$ Tris, $0.5 \%$ deoxycholic acid, $1 \%$ Triton X-100, $0.5 \%$ SDS, 25 mM EDTA, pH 8.0, supplemented by protease inhibitor (Complete, Roche) and phosphatase inhibitor (PhosSTOP, Roche)). The RIPA insoluble pellet after centrifugation at $50,000 \times \mathrm{g}$ for $20 \mathrm{~min}$ was solubilized with $70 \%$ formic acid [21]. Tau levels in these fractions were measured by various tau ELISAs described below. Human tau was detectable in both RAB and RIPA fractions, thus total tau levels in both fractions were used to calculate the half-life of "intracellular soluble tau". Tau in $70 \%$ formic acid fractions was defined as the "intracellular insoluble tau". We observed high background values when we measured phosphorylated tau in RIPA fractions with our assays, thus only RAB fractions were used as soluble fractions to reliably measure soluble phosphorylated tau levels. To confirm the specificity of ELISAs used in this study, hippocampus from tau knockout mice, wildtype mice and hTau mice were homogenized with $\mathrm{RAB}$ buffer and analyzed.

\section{Tau ELISAs}

Absolute levels of human and murine tau in ISF and brains were measured by ELISAs which use a combination of Tau5/biotinylated HT7 (HT7B) and HJ9.2/ biotinylated HJ8.7 (HJ8.7B) monoclonal anti-tau antibodies respectively as previously described [20]. Relative levels of human tau phosphorylated at S202/T205 and T231/S235 were detected by in house ELISAs with a combination of HJ8.5 [28]/biotinylated AT8 (Pierce) and HJ8.5/biotinylated AT180 (Pierce) respectively. To measure total (both murine and human) phosphorylated tau at S202/T205, combinations of HJ8.7/biotinylated AT8 were used. HJ8.5 recognizes human tau specifically whereas HJ8.7 has an identical affinity toward murine and human tau. Human phosphorylated tau at S396 was measured by Tau [pS396] Human ELISA kit (Life technologies). For phosphorylated tau measurement using in house phosphorylated tau ELISAs, RAB soluble fractions from a 7 month old P301S human tau transgenic mouse was used as a standard. Thus, instead of absolute levels, relative levels of phosphorylated tau normalized to "0 day group" were used for half-life calculation.

\section{Lactate measurements in ISF}

ISF lactate levels were determined with a YSI2700 biochemistry analyzer (YSI Life Sciences), which quantifies their levels with immobilized enzyme biosensors specific for the substrates [20].

\section{The calculation of half-life}

The half-life of tau was calculated as we previously reported using the slope of linear regression that includes all data points $\left(t_{1 / 2}=0.693 / k\right.$, where $\left.k=2.303 \quad \mathrm{k}^{\prime}\right)$ (Fig. 1c) $[20,23]$. In order to consider the time required for transgene switch off in our strategy, we measured luciferase activity [6]. Following doxycycline treatment, bioluminescence signal intensity by luciferase was constant for $4 \mathrm{~h}$ and then started to decline (unpublished observation of $\mathrm{KH}$ and EMM). This 4 h-delay time consists of less than $5.3 \%$ of the half-life of any form of tau assessed in this study, suggesting that the contribution of this delay to halflife calculation is negligible.

\section{Statistical analysis}

Data in figures represent mean \pm s.e.m. The comparison of multiple groups was done by one-way ANOVA with Tukey's post hoc test. Correlation coefficients $\left(R^{2}\right)$ were calculated to see the goodness of fit for the linear regression. To examine statistically significant differences in turnover rates, the slope of linear regression that includes all data points over time were compared using analysis of covariance (ANCOVA) [31].

\section{Abbreviations}

AD: Alzheimer's disease; CSF: Cerebrospinal fluid; NFTs: Neurofibrillary tangles; HJ8.7B: (Biotinylated HJ8.7); HT7B: (Biotinylated HT7); ISF: Brain interstitial fluid; RAB: Reassembly buffer.

\section{Competing interests}

$\mathrm{DMH}$ and Washington University have ownership interests in C2N Diagnostics and Washington University has licensed the use of certain anti-tau antibodies for potential therapeutic use to C2N Diagnostics. These antibodies have been licensed by $\mathrm{C} 2 \mathrm{~N}$ to AbbVie. $\mathrm{DMH}$ is not doing research with humanized tau antibodies. $\mathrm{DMH}$ is on the scientific advisory board of C2N Diagnostics, Neurophage, and Denali, consults for AstraZeneca, Genentech, and Eli Lilly, and his laboratory receives research grants from C2N Diagnostics, AbbVie, and Eli Lilly. The remaining authors declare no competing financial interests.

\section{Authors' contributions}

KY and DMH conceived and designed the experiments. KY performed all microdialysis experiments, brain extraction and ELISAs. TKP performed some phosphorylated tau ELISA experiments. TKP and KH acquired the data not included in the manuscript. TEM, HJ, and FRS collected tissue samples. $\mathrm{KY}$ and $\mathrm{DMH}$ analyzed the data. $\mathrm{KH}$ and EMM provided the mice and assisted with data interpretation. KY, EMM and DMH wrote the manuscript. All authors read and approved the final version of the manuscript.

\section{Acknowledgements}

This work was supported by Japan Society for the promotion of Science to KY and the Tau Consortium to DMH and EMM. We would like to thank Dr. Timothy M. Miller and Dr. Sarah L. DeVos in Washington University for providing us with hTau mice.

\section{Author details}

'Department of Neuropathology, Graduate School of Medicine, The University of Tokyo, Tokyo 113-0033, Japan. ²Department of Neurology, Hope Center for Neurological Disorders, Knight Alzheimer's Disease Research Center, Washington University School of Medicine, St. Louis, Missouri, 63110, USA. ${ }^{3} \mathrm{MPI}$ for Neurological Research, Hamburg Outstation, c/o DESY, Notkestr. 85, 22607 Hamburg, Germany. ${ }^{4}$ DZNE (German Ctr Neurodegen. 
Diseases), Ludwig-Erhard-Allee 2, 53175 Bonn, Germany. ${ }^{5}$ CAESAR Research Center, Ludwig-Erhard-Allee 2, 53175 Bonn, Germany.

Received: 22 June 2015 Accepted: 21 October 2015 Published online: 26 October 2015

\section{References}

1. De Calignon A, Polydoro M, Suárez-Calvet M, William C, Adamowicz DH, Kopeikina KJ, et al. Propagation of tau pathology in a model of early Alzheimer's disease. Neuron. 2012;73:685-97.

2. Clavaguera F, Bolmont T, Crowther RA, Abramowski D, Frank S, Probst A, et al. Transmission and spreading of tauopathy in transgenic mouse brain. Nat Cell Biol. 2009;11:909-13.

3. Frost $B$, Jacks RL, Diamond MI. Propagation of tau misfolding from the outside to the inside of a cell. J Biol Chem. 2009;284:12845-52.

4. Liu L, Drouet V, Wu JW, Witter MP, Small SA, Clelland C, et al. Trans-synaptic spread of tau pathology in vivo. PLoS One. 2012;7:e31302.

5. Iba M, Guo JL, McBride JD, Zhang B, Trojanowski JQ, Lee VM-Y. Synthetic tau fibrils mediate transmission of neurofibrillary tangles in a transgenic mouse model of Alzheimer's-like tauopathy. J Neurosci. 2013;33:1024-37.

6. Van der Jeugd A, Hochgräfe K, Ahmed T, Decker JM, Sydow A, Hofmann A, et al. Cognitive defects are reversible in inducible mice expressing pro-aggregant full-length human Tau. Acta Neuropathol. 2012;123:787-805.

7. Roberson ED, Scearce-Levie K, Palop JJ, Yan F, Cheng IH, Wu T, et al. Reducing endogenous tau ameliorates amyloid beta-induced deficits in an Alzheimer's disease mouse model. Science. 2007;316(80-):750-4

8. Santacruz K, Lewis J, Spires T, Paulson J, Kotilinek L, Ingelsson M, et al. Tau suppression in a neurodegenerative mouse model improves memory function. Science. 2005;309:476-81.

9. David DC, Layfield R, Serpell L, Narain Y, Goedert M, Spillantini MG Proteasomal degradation of tau protein. J Neurochem. 2002;83:176-85.

10. Lim J, Balastik M, Lee TH, Nakamura K, Liou Y, Sun A, et al. Pin1 has opposite effects on wild-type and P301L tau stability and tauopathy. J Clin Invest. 2008;118:1877-89

11. Carrettiero DC, Hernandez I, Neveu P, Papagiannakopoulos T, Kosik KS. The cochaperone BAG2 sweeps paired helical filament- insoluble tau from the microtubule. J Neurosci. 2009;29:2151-61.

12. Min S-W, Cho S-H, Zhou Y, Schroeder S, Haroutunian V, Seeley WW, et al. Acetylation of tau inhibits its degradation and contributes to tauopathy. Neuron. 2010;67:953-66.

13. Dickey CA, Kamal A, Lundgren K, Klosak N, Bailey RM, Dunmore J, et al. The high-affinity HSP90-CHIP complex recognizes and selectively degrades phosphorylated tau client proteins. J Clin Invest. 2007;117:648-58.

14. Shimura H, Schwartz D, Gygi SP, Kosik KS. CHIP-Hsc70 complex ubiquitinates phosphorylated tau and enhances cell survival. J Biol Chem. 2004;279:4869-76.

15. Hochgräfe K, Sydow A, Mandelkow E-M. Regulatable transgenic mouse models of Alzheimer disease: onset, reversibility and spreading of Tau pathology. FEBS J. 2013;280:4371-81.

16. Eckermann K, Mocanu M-M, Khlistunova I, Biernat J, Nissen A, Hofmann A, et al. The beta-propensity of Tau determines aggregation and synaptic loss in inducible mouse models of tauopathy. J Biol Chem. 2007;282:31755-65.

17. Von Bergen M, Barghorn S, Li L, Marx A, Biernat J, Mandelkow E-M, et al. Mutations of Tau protein in frontotemporal dementia promote aggregation of paired helical filaments by enhancing local \{beta\}-structure. J Biol Chem. 2001;276:48165-74.

18. Van Swieten JC, Bronner IF, Azmani A, Severijnen L-A, Kamphorst W, Ravid $\mathrm{R}$, et al. The DeltaK280 mutation in MAP tau favors exon 10 skipping in vivo. J Neuropathol Exp Neurol. 2007;66:17-25.

19. Momeni P, Pittman A, Lashley T, Vandrovcova J, Malzer E, Luk C, et al. Clinical and pathological features of an Alzheimer's disease patient with the MAPT delta K280 mutation. Neurobiol Aging. 2009;30:388-93.

20. Yamada K, Holth JK, Liao F, Stewart FR, Mahan TE, Jiang H, et al. Neuronal activity regulates extracellular tau in vivo. J Exp Med. 2014;211:387-93.

21. Yamada K, Cirrito JR, Stewart FR, Jiang H, Finn MB, Holmes BB, et al. In vivo microdialysis reveals Age-dependent decrease of brain interstitial fluid Tau levels in P301S human Tau transgenic mice. J Neurosci. 2011:31:13110-7.

22. Andorfer C, Kress Y, Espinoza M, De Silva R, Tucker KL, Barde $Y$-A, et al. Hyperphosphorylation and aggregation of tau in mice expressing normal human tau isoforms. J Neurochem. 2003;86:582-90.
23. Castellano JM, Kim J, Stewart FR, Jiang H, DeMattos RB, Patterson BW, et al. Human apoE isoforms differentially regulate brain amyloid- $\beta$ peptide clearance. Sci Transl Med. 2011;3:89ra57.

24. Poppek D, Keck S, Ermak G, Jung T, Stolzing A, Ullrich O, et al. Phosphorylation inhibits turnover of the tau protein by the proteasome: influence of RCAN1 and oxidative stress. Biochem J. 2006:400:511-20.

25. Ling S-C, Albuquerque CP, Han JS, Lagier-Tourenne C, Tokunaga S, Zhou H, et al. ALS-associated mutations in TDP-43 increase its stability and promote TDP-43 complexes with FUS/TLS. Proc Natl Acad Sci U S A. 2010;107:13318-23.

26. Li W, Lesuisse C, Xu Y, Troncoso JC, Price DL, Lee MK. Stabilization of alpha-synuclein protein with aging and familial parkinson's disease-linked A53T mutation. J Neurosci. 2004;24:7400-9.

27. Price JC, Guan S, Burlingame A, Prusiner SB, Ghaemmaghami S. Analysis of proteome dynamics in the mouse brain. Proc Natl Acad Sci U S A 2010;107:14508-13.

28. Yanamandra $K$, Kfoury $N$, Jiang $H$, Mahan TE, Ma S, Maloney SE, et al. Anti-tau antibodies that block tau aggregate seeding in vitro markedly decrease pathology and improve cognition in vivo. Neuron. 2013;80:402-14.

29. Yoshiyama Y, Higuchi M, Zhang B, Huang SM, Iwata N, Saido TC, et al. Synapse loss and microglial activation precede tangles in a P301S tauopathy mouse model. Neuron. 2007;53:337-51.

30. Dawson HN, Ferreira A, Eyster MV, Ghoshal N, Binder LI, Vitek MP. Inhibition of neuronal maturation in primary hippocampal neurons from tau deficient mice. J Cell Sci. 2001;114(Pt 6):1179-87.

31. Basak JM, Kim J, Pyatkivskyy $Y$, Wildsmith KR, Jiang $H$, Parsadanian $M$, et al Measurement of apolipoprotein $\mathrm{E}$ and amyloid $\beta$ clearance rates in the mouse brain using bolus stable isotope labeling. Mol Neurodegener. 2012;7:14.

\section{Submit your next manuscript to BioMed Central and take full advantage of:}

- Convenient online submission

- Thorough peer review

- No space constraints or color figure charges

- Immediate publication on acceptance

- Inclusion in PubMed, CAS, Scopus and Google Scholar

- Research which is freely available for redistribution

Submit your manuscript at www.biomedcentral.com/submit
C Biomed Central 